\title{
Effect of Changes in Neurofilament Content on Caliber of Small Axons: The $\beta, \beta^{\prime}$-Iminodipropionitrile Model
}

\author{
Irma M. Parhad, ${ }^{1}$ Arthur W. Clark, ${ }^{2}$ and John W. Griffin ${ }^{3}$ \\ 1 Department of Pathology and Clinical Neurosciences and 'Department of Pathology, University of Calgary, Calgary, \\ Alberta, T2N 4N1, Canada, and 'Department of Neurology and Neuroscience, Johns Hopkins University, Baltimore, \\ Maryland
}

\begin{abstract}
The structural role of neurofilaments in the normal axon and the consequences of altered axonal transport of neurofilaments have been extensively studied in large axons. These studies suggest that neurofilament numbers and interneurofilament spacing are major determinants of axonal crosssectional area. In contrast, in small axons and dendrites, microtubules and membranous organelles appear to be the most closely correlated with size and shape of the cell process.
\end{abstract}

In this study we have examined the effect of impairment in neurofilament transport on small axons, typical of most CNS pathways. Neurofilament transport was impaired by administration of $\beta, \beta^{\prime}$-iminodipropionitrile (IDPN), resulting in proximal accumulation and distal depletion of neurofilaments. The evolution of these changes was studied in the optic nerves of guinea pigs treated with IDPN, 1-35 weeks following intoxication. The effect of this redistribution of neurofilaments on cross-sectional area of small axons was evaluated using quantitative ultrastructural methods. Our results show that with the alteration in neurofilament transport seen with IDPN intoxication, there is a wide spectrum of neurofilament densities, ranging from a 5 -fold increase above normal in the proximal axon, to a 5-fold decrease below normal in the distal axon. Although the optic nerve fibers enlarge with the increase in neurofilament content, they do not atrophy significantly with the continued loss of neurofilaments. We conclude that factors other than neurofilament content are capable of maintaining size and shape of these small axons. Candidate organelles include microtubules and membranous organelles and possibly other axonal elements.

Neurofilament (Nf) content has been correlated with cross-sectional area (CSA) of axons in the PNS and CNS (Friede and Samorajski, 1970; Friede et al., 1971; Smith, 1973) in unmyelinated and myelinated fibers (Friede et al., 1971; Berthold, 1978) and in various regions of the same axon (Bcrthold, 1978). These studies have shown that under normal conditions $\mathrm{Nf}$ density remains relatively stable and therefore the CSA changes pro-

Received Oct. 15, 1986; revised Jan. 12, 1987; accepted Jan. 20, 1987.

We wish to thank Drs. Jeffrey Rosenfeld, Alfred Rademaker, and Mark Bisby for helpful discussions. I.M.P. is a recipient of Alberta Heritage Foundation for Medical Research Scholarship.

Correspondence should be addressed to Irma M. Parhad, M.D., Rm. 2040, Health Sciences Building, 3300 Hospital Drive, N.W., Calgary, Alberta T2N 4N I, Canada.

Copyright (C) 1987 Society for Neuroscience $0270-6474 / 87 / 072256-08 \$ 02.00 / 0$ portionally to the Nf content of an axon (Friede et al., 1971; Berthold, 1978).

Nfs consist of 3 proteins (Hoffman and Lasek, 1975), which are synthesized in the cell body and rapidly assembled into stable polymers (Lasek et al., 1984). They are delivered to the axon in the slow component of axonal transport (Hoffman and Lasek, 1975; Black and Lasek, 1980). Although some modification of Nf proteins does occur in the axon (Nixon et al., 1982; Nixon and Logvinenko, 1986), actual degradation of Nfs occurs for the most part in the axonal terminal (Roots, 1983). These observations suggest that processes that alter the transport of $\mathrm{Nf}$ proteins can significantly influence the $\mathrm{Nf}$ content and caliber of axons.

In a series of studies on regenerating and developing axons of the sciatic motor complex, Hoffman et al. (1984, 1985a, b) have shown that the axonal caliber changes with the amount of $\mathrm{Nf}$ protein delivered. In these models, Nf density remains constant, so that a decrease in Nf number results in a proportional reduction in CSA; this occurs without a change in perimeter, so that the axon becomes less circular (Hoffman et al., 1984; Dyck et al., 1985). Such data suggest that axonal size and shape follow $\mathrm{Nf}$ content in a passive fashion. The sciatic motor complex, however, has large, Nf-rich fibers, which are not representative of most of the fibers of the nervous system.

In this study, we have examined the effect of impairment in Nf transport on the axons of the guinea pig optic nerve. This system was chosen because of its relatively small myelinated fibers, typical of most of the central nervous system fibers. $\mathrm{Nf}$ transport was impaired by administration of $\beta, \beta^{\prime}$-iminodipropionitrile (IDPN). IDPN is an agent that impairs the rate of transport of $\mathrm{Nf}$ triplet proteins, resulting in a redistribution of $\mathrm{Nfs}$ with excessive accumulation in the proximal part of the axon and depletion in the rest of the axon (Griffin et al., 1978, 1985; Clark et al., 1980; Parhad et al., 1982, 1986). In previous studies of large axons of the PNS, this maldistribution of Nfs was manifestcd by proximal axonal swelling and distal axonal atrophy (Clark et al., 1980). The relationship between the $\mathrm{Nf}$ accumulation and axonal swelling, on the one hand, and the Nf depletion and the atrophy, on the other, has not been examined in the small fibers. This study systematically examined the changes in Nf density and content, as well as content of microtubules (Mt), membranous organelles and mitochondria (MOM), in relation to changes in CSA at various times following IDPN intoxication. The results indicate that while $\mathrm{Nfs}$ and inter-Nf distance are closely correlated with axonal caliber in normal fibers, when Nf delivery is impaired the density can be markedly reduced. In other words, Nf content can be reduced out of pro- 
portion to CSA. These observations indicate that, at least in small fibers, other cellular organelles are able to maintain the shape and size of the cell process.

\section{Materials and Methods}

\section{Experimental design}

Female young adult (approximately 7-week-old, 400-600 gm) albino guinea pigs, Hartley strain (Buckberg Lab Animals, Rockland, NY), were given an intraperitoneal dose of $0.3 \mathrm{gm} / \mathrm{kg}$ of IDPN (Eastman Kodak Company, Rochester, NY) and mainlained on $0.02 \%$ IDPN in drinking water. One intoxicated animal and one age-matched control were killed $1,2,5$, and 35 weeks after intoxication, and the visual system was processed for morphologic and morphometric studies.

\section{Tissue fixation preparation for morphologic/morphometric studies}

Animals were anesthetized with chloral hydrate solution $(400 \mathrm{mg} / \mathrm{kg}$ intraperitoneally) and gravity-perfused through the ascending aorta with a rinse $(100 \mathrm{ml})$ of $0.1 \mathrm{~m}$ phosphate buffer $(\mathrm{pH} 7.3$, room temperature), followed by 1 liter of $5 \%$ glutaraldehyde in $0.1 \mathrm{~m}$ phosphate buffer $(\mathrm{pH}$ 7.3 , room temperature). The visual system was dissected in toto (retina and optic pathway to the point of entry into the lateral geniculate body) and measured. The prelaminar (proximal) optic nerve segment, from a region in midretina to the lamina cribrosa, is approximately $1.5 \mathrm{~mm}$ in length. The postlaminar (distal) segment from the lamina cribrosa to the lateral geniculate body is approximately $18 \mathrm{~mm}$ (Fig. 1). Tissue samples were taken from the retina, optic disk region, and optic nerve cut transversely at $2-4 \mathrm{~mm}$ and $8-10 \mathrm{~mm}$ distal to the lamina cribrosa (Fig. 1). Tissue samples were washed in $0.1 \mathrm{~m}$ phosphate buffer, postfixed in $2 \%$ osmium tetroxide, dehydrated in a graded series of ethanols, cleared with propylene oxide, and infiltrated with Araldite (Lab Research, Burlington, VT). Thick sections $(1-2 \mu \mathrm{m})$ were stained with toluidine blue. Ultrathin sections were stained with uranyl acetate and lead citrate and examined in a Hitachi H-600 TEM electron microscope.

\section{Measurement of axonal CSA and shape factor}

Prelaminar optic nerve. Because a true cross section for all axons cannot be obtained in the same section in the optic disk region, only those axons in cross section were measured. In the IDPN-intoxicated animals, electron micrographs $(\times 12,500)$ of 4 nonoverlapping fields were taken from this region, and 200 randomly selected appropriately oriented fibers were measured in each animal. In the control group, the best cross section of the optic disk was seen in the 42-week-old animal; electron micrographs $(\times 12,500)$ of 8 nonoverlapping fields were taken from this optic disk section and 500 axons measured.

Postlaminar optic nerve. Measurement of axonal CSA and circumference were done at 2 levels $(2-4$ and $8-10 \mathrm{~mm}$ ) distal to the lamina cribrosa. At each level, electron micrographs $(\times 12,500)$ of 3 nonoverlapping fields were taken, and all myelinated axons (except those sectioned through nodes of Ranvier or paranodal regions or clearly cut in oblique section) were measured. At each level, 200 axons (400 axons/ animal) were measured. The CSA and circumference of axons were measured, and the shape factor $\left(4 \pi\right.$ area/circumference $\left.{ }^{2}\right)$ determined using a HI-pad digitizer (Houston Instrument) interphased with a computer (Apple II + or IBM-PC AT) using Bioquant II program (R\&M Biometrics, Nashville, TN).

\section{Quantitation of axons in optic nerves}

To evaluate the possibility of loss of fibers in the optic pathway following IDPN intoxication, the numbers of optic nerve fibers in control and intoxicated animals were calculated. The entire cross sections of each optic nerve (2-4 $\mathrm{mm}$ distal to the lamina cribrosa) were photographed $(\times 510)$ and the area digitized. Approximately $1 \%$ of the CSA of the optic nerve was photographed $(\times 12,500)$ using the EM. All myelinated axons in these regions were counted and the total number of myelinated fibers in each optic nerve obtained.

\section{Quantitation of organelle profiles}

Randomly selected axons were photographed $(\times 87,500-150,000)$ for counting $\mathrm{Nf}$ and $\mathrm{Mt}$ profiles, for measuring inter-Nf distance, and for evaluating the relative area occupied by MOM. All Mts and Nfs in each

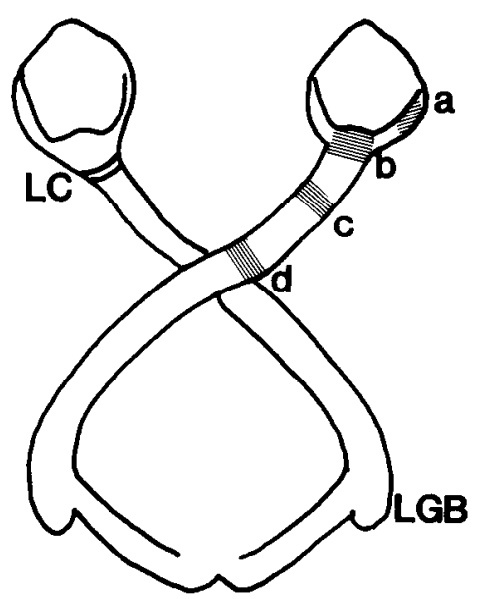

Figure 1. Diagram of the visual system in adult guinea pigs: The lamina cribrosa $(L C)$ and the lateral geniculate body $(L G B)$ are marked. The stippled areas are regions sampled for morphologic and morphometric analyses: $(a)$ retina, $(b)$ optic disk, and optic nerve $(c)$ 2-4 $\mathrm{mm}$ and $(d)$ $8-10 \mathrm{~mm}$ distal to the lamina cribrosa.

of these axons were counted, except for axons with a CSA $>3 \mu \mathrm{m}^{2}$. In these axons the Nfs were counted in an area of $1 \mu \mathrm{m}^{2}$. All counts were done on randomly assorted photomicrographs by a single observer. Nfs and $\mathrm{Mt}$ in the same axon counted on separate occasions varied by less than $3 \%$. The area occupied by MOM was digitized and expressed as a percentage of the CSA of axons. A total of 61 control and 90 intoxicated axons was evaluated for $\mathrm{Nf}$ and $\mathrm{Mt}$ densities and for the area occupied by MOM. Nf and Mt content in an axon was estimated using the figures in Tables 1-3. Inter-Nf distance, the distance between the centers of 2 adjacent $\mathrm{Nf}$ profiles in cross section within a group, were measured. One hundred such distances in 3-5 axons were measured in each instance, using electron micrographs (final magnification $\times 325,000$ ).

\section{Statistical analysis}

The CSA of axons in the optic nerves of control and IDPN-intoxicated animals display a unimodal distribution (Parhad et al., 1986). One- and two-way analyses of variance (ANOVA-1, ANOVA-2) followed by Duncan multiple-range test (Duncan, 1955) were performed to test thc effects of position along the axon, age of animals, and duration of intoxication on the CSA.

Regression analyses were performed to test the relationship between $\mathrm{Nf}$ and Mt density, and area occupied by MOM, on the one hand, and axonal CSA, on the other, for IDPN and control animals. The $r$ test (Snedecor and Cochran, 1980) was used to test for significance of the regression line, the straight line regression model (Kleinbaum and Kupper, 1978) to test for the equality of the slopes, and Student's $t$ test to compare the difference between 2 means. All statistical analyses were performed on Bioquant II using an IBM-PC AT computer. All results are expressed as means \pm SEM.

\section{Results}

\section{Control}

The prelaminar (proximal) axons in the albino guinea pigs are unmyelinated, whereas the postlaminar (distal) optic nerve axons are largely myelinated (Fig. 2, $a, b$ ). There is no difference in the CSA of axons measured at different levels of the optic nerve (prelaminar versus postlaminar: ANOVA-1, $p>0.05 ; 2-$ 4 vs $8-10 \mathrm{~mm}$ distal to the lamina cribrosa: ANOVA-2, $p>$ 0.05; Tables 1 and 2). The CSA of postlaminar axons remains unchanged between the ages of 9 of 42 weeks (ANOVA-2, $p>$ 0.25 ); the 8-week-old animal has slightly smaller axons (ANOVA-2, $p<0.01$; Duncan's, $p<0.01$; Table 2).

The Nf density $(77.8 \pm 4.1)$ does not vary as a function of the axonal CSA (Table 3: correlation coefficient $(r),-0.003 ; n$, 

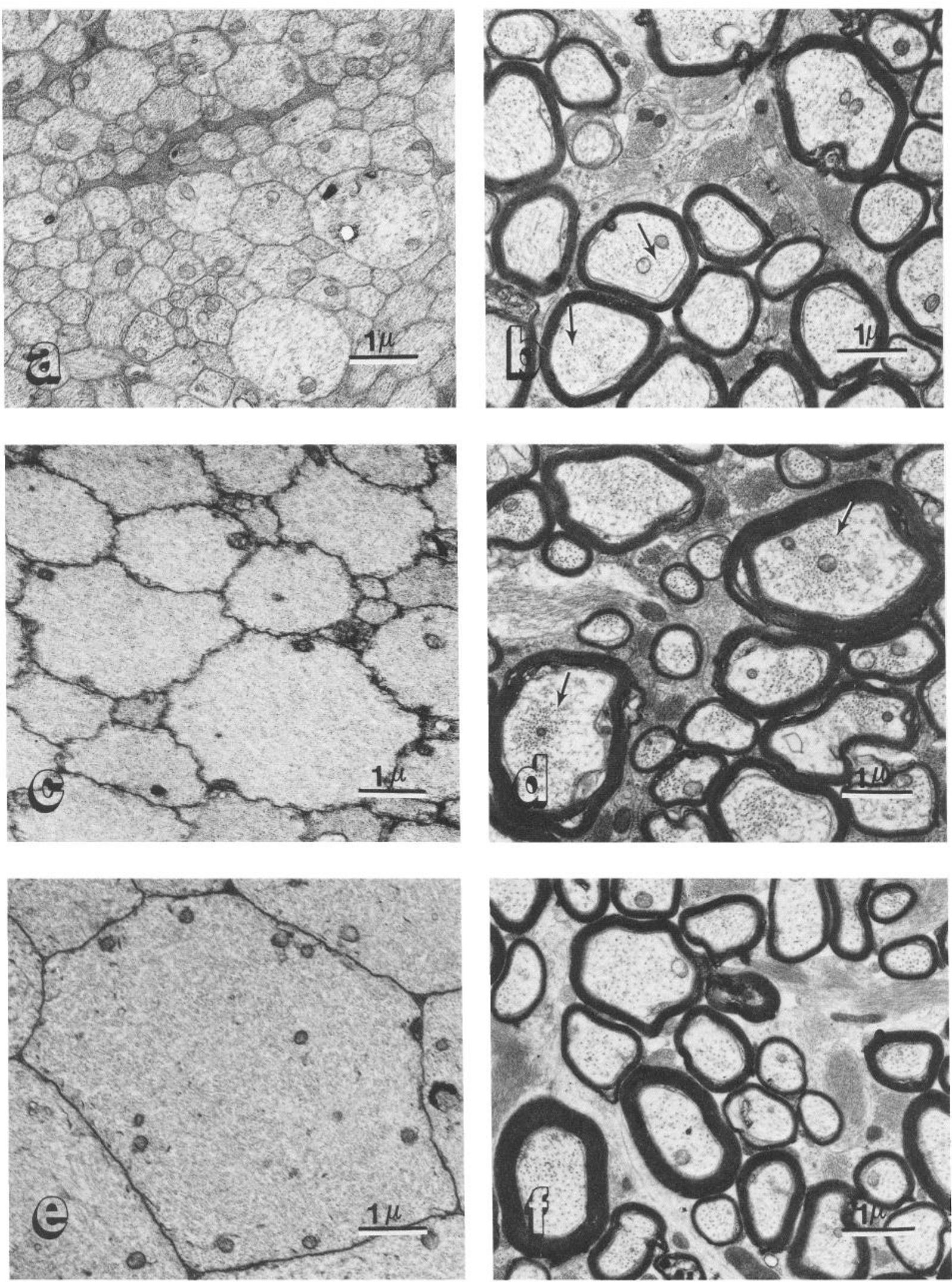

Figure 2. Electron micrographs of axons in the visual system. $a$ and $b$, Prelaminar unmyelinated and postlaminar myelinated axons, respectively, from a control animal, with normal grouped distribution of Nfs (arrows). $c$ and $d$, Pre- and postlaminar axons, respectively, from an animal intoxicated with IDPN for 2 weeks. Note the increase in Nf density and CSA in the prelaminar axons and the segregation of Nf from Mt, the Mt forming a central grouping (arrows) in the postlaminar axons. $e$ and $f$, Pre- and postlaminar axons, respectively, in an animal intoxicated for 35 weeks. Note the massive enlargement in the CSA of the prelaminar axons, whereas the $\mathrm{Nf}$ density is the same as in $c$. In the postlaminar axons, there is a marked depletion of Nf. 


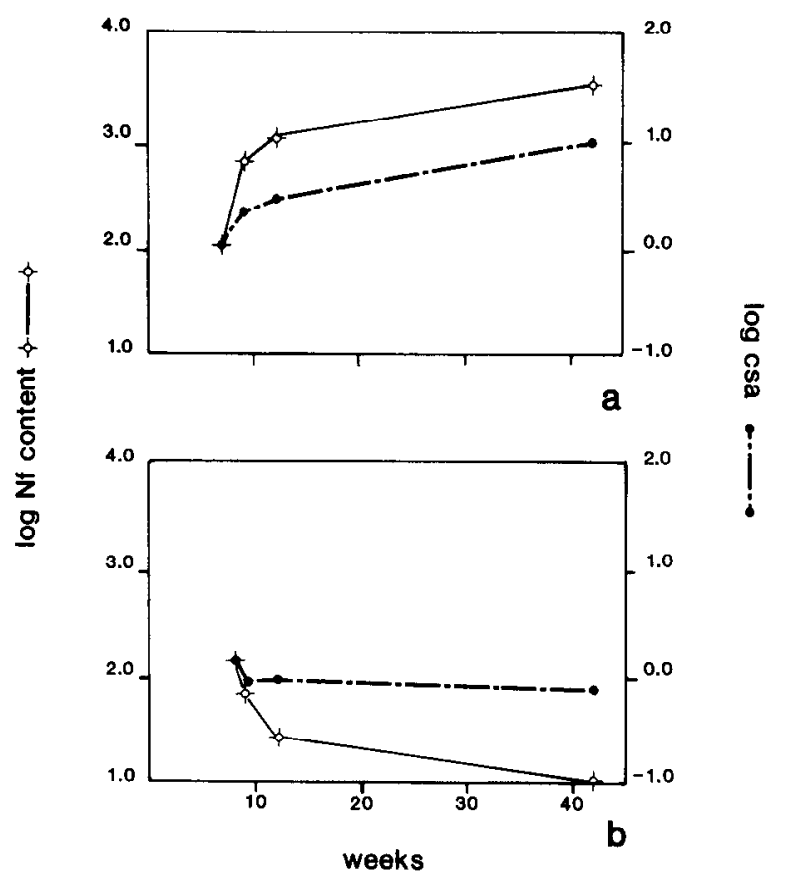

Figure 3. Nf content and CSA as functions of the duration of intoxication. The ordinates are the Nf content (mean Nf density $x$ mean CSA) and mean CSA $\left(\mu \mathrm{m}^{2}\right)$. The data are graphed on a semilogarithmic scalc. $a$, Proximal axons. Notc the increase in Nf content, which, early on, exceeds the increase in CSA, resulting in an increase in Nf density. Once the new Nf density is reached,the CSA increases in parallel with the $\mathrm{Nf}$ content. $b$, Distal axons. Note the decrease in $\mathrm{Nf}$ content exceeds the decrease in CSA, resulting in a decrease in Nf density. The CSA does not continue to decrease with the duration of intoxication.

35 ; slope, $y=77.9-0.059 x ; p>0.5)$. The Mt density correlates inversely with the axonal CSA $(r,-0.78 ; n, 61$; slope, $y=1.97-$ $0.22 x ; p<0.001-$ scc Fig. 5). The area occupied by MOM is less than $6 \%$ of the CSA of axons (Table 3). The Nf and Mt densities and the relative area occupied by MOM are the same in the pre- and postlaminar axons (Table 3, Student's $t$ tests, $p>0.05$ ).

\section{Evolution of changes with IDPN intoxication}

The earliest change seen following IDPN intoxication is the disorganization of the cytoskeletal elements consisting of a segregation of $\mathrm{Mt}$ from Nfs. This change is evident in both the preand postlaminar axons 1 week following intoxication (not shown). In the prelaminar axons it is rapidly supplanted by the massive accumulation of $\mathrm{Nfs}$ (Fig. 2, $c, e$ ). In the postlaminar axons it becomes more prominent at 2 and 5 weeks after intoxication

Table 1. CSA of prelaminar axons in control and IDPN animals

\begin{tabular}{|c|c|c|}
\hline \multirow{2}{*}{$\begin{array}{l}\text { Age } \\
\text { (weeks) }\end{array}$} & \multicolumn{2}{|l|}{$\mathrm{CSA}\left(\mu \mathrm{m}^{2}\right)$} \\
\hline & Control $^{a}$ & IDPN $^{b}$ \\
\hline 9 & - & $1.97 \pm 0.31$ \\
\hline 12 & - & $2.55 \pm 0.10$ \\
\hline 42 & $1.18 \pm 0.05$ & $8.85 \pm 1.07$ \\
\hline
\end{tabular}

Means \pm SE given.

a 200 axons measured

${ }^{b} 500$ axons measured at each time point.

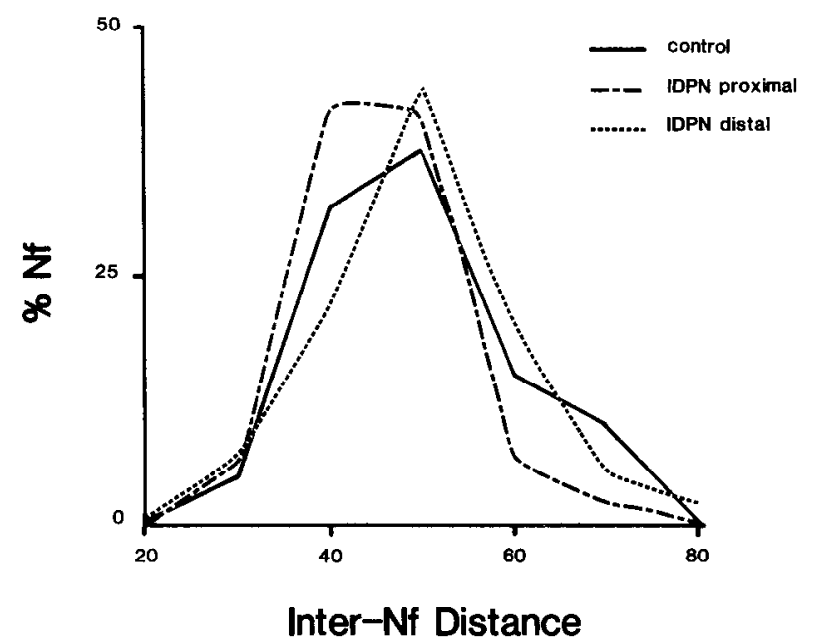

Figure 4. Inter-Nf distances in control and IDPN proximal and distal axons. The Nfs are only slightly closer together in the proximal axon following IDPN intoxication $(39.26 \pm 0.86 \mu \mathrm{m})$ than in control $(44.07 \pm$ $1.01 \mu \mathrm{m})$ or in the distal axon following intoxication $(45.87 \pm 0.96 \mu \mathrm{m})$. Student's $t$ tests, $p<0.001$.

(Fig. $2 d$ ) but disappears when the Nfs become markedly depleted (Fig. 2f; Table 3).

In the proximal axon there is a progressive increase in the $\mathrm{Nf}$ content, and axonal CSA (Figs. 2, $a, c, e, 3 a$; Table 1). In the early stages, in any axon the increase in Nf content exceeds that of the axonal CSA, resulting in a marked increase in Nf density from approximately 70 to $400 \mathrm{Nf} / \mu \mathrm{m}^{2}$. With continued intoxication, more axons seem to attain this density (Table 3). When the new $\mathrm{Nf}$ density is reached, the CSA again increases in proportion to the Nf content (Fig. $3 a$ ).

In the distal axon, there is a mild but significant atrophy (30\%) in the CSA as compared with the control (Fig. 2, $b, d$, and $f$, and Table 2; ANOVA-2, $p<0.01$ ). This atrophy is first seen 2 weeks following intoxication, occurs simultaneously and equally along the axon, and does not progress with the duration of intoxication (Table 2: ANOVA-2, $p>0.25$ ). The circumference of the axons changes as well, and the axons maintain the same circularity (shape factor control: $0.824 \pm 0.006$; IDPN 35 weeks, $0.826 \pm 0.005 ; p>0.5)$.

Table 2. Comparison of axonal CSA in 2 postlaminar regions in control and IDPN animals

\begin{tabular}{lll} 
& \multicolumn{2}{c}{$\operatorname{CSA}\left(\mu \mathrm{m}^{2}\right)$} \\
\cline { 2 - 3 } Group & $2-4 \mathrm{~mm}^{\alpha}$ & $8-10 \mathrm{~mm}^{a}$ \\
\hline Control & $0.90 \pm 0.04^{c}$ & $0.91 \pm 0.05$ \\
8 wk $(400)^{h}$ & $1.22 \pm 0.07$ & $1.06 \pm 0.06$ \\
9 wk (550) & $1.03 \pm 0.07$ & $1.24 \pm 0.06$ \\
12 wk (632) & $1.05 \pm 0.06$ & $1.25 \pm 0.07$ \\
42 wk (930) & & \\
IDPN & $1.03 \pm 0.06$ & $0.99 \pm 0.05$ \\
8 wk (400) & $0.85 \pm 0.06$ & $0.70 \pm 0.03$ \\
9 wk (500) & $0.86 \pm 0.05$ & $0.84 \pm 0.05$ \\
12 wk (320) & $0.72 \pm 0.03$ & $0.79 \pm 0.04$ \\
42 wk (680) &
\end{tabular}

${ }^{a}$ Distance from the lamina cribrosa.

${ }^{b}$ Weight of animal when killed (in grams).

${ }^{c}$ Means \pm SE given; 200 axons were measured in each group. 


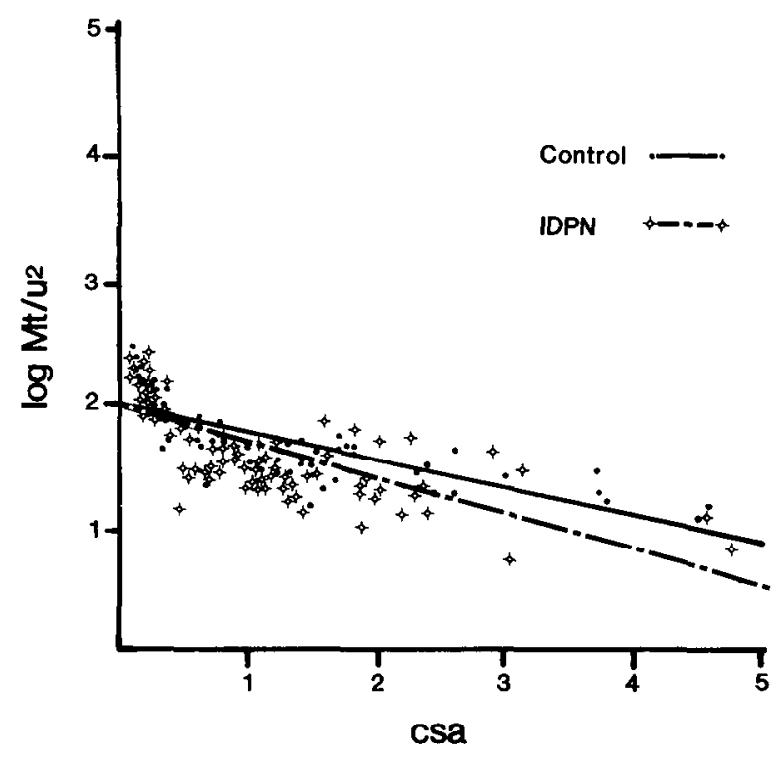

Figure 5. Mt density as a function of CSA in control and intoxicated animals. The slope is similar in control and IDPN axons $(p>0.5)$. Slope for control: $y=1.97-0.22 x, n=61, r=0.78, p<0.001$; IDPN: $y=1.97-0.29 x, n=81, r=0.73, p<0.001$.

This axonal atrophy is not due to selective loss of large fibers. The total number of myelinated fibers in intoxicated optic nerves is no different from that in control (IDPN: 130,993 \pm 6009 , $n=3$; control: $142,275 \pm 5264, n=3$; Student's $t$ test, $p>$ $0.05)$, and wallerian-like degeneration is rarely seen.

In the distal axon, there is a continuous decline in Nf content, which results in a marked decline (80\%) in Nf density (Figs. $2 f$ and $3 b$; Tables 2 and 3 ).

Although the density of Nfs increases in the proximal axon, and decreases in the distal axon following IDPN intoxication, the inter-NF distance remains relatively constant (Fig. 4). The Mt density (Fig. 5) and the relative area occupied by MOM (Table 3; Student's $t$ test, $p>0.05$ ) also remain unchanged following IDPN intoxication.

In the distal axons following IDPN intoxication, the CSA does not change as a function of $\mathrm{Nf}$ content (Fig. $6 \mathrm{a}$ ). In contrast, the CSA correlates directly with the Mt content and area occupied by MOM (Fig. 6, $b, c$ ).

\section{Selective vulnerability of axons to IDPN}

Assuming that the largest preintoxication axons become the largest axons following intoxication, then the relative increase in the large axons is greater than in the small axons (Fig. 7). In the distal axons, however, preintoxication large and small axons have the same relative degree of atrophy (Fig. 7).

\section{Discussion}

\section{Effect of IDPN on Nf transport}

IDPN primarily affects the rate of $\mathrm{Nf}$ protein transport (Griffin et al., 1985; Parhad et al., 1986). Consequently, the effect of IDPN on axonal transport varies depending on the $\mathrm{Nf}$ content of the system (Griffin et al., 1985; Parhad et al., 1986). Its effect on the Nf-rich large axons of the sciatic motor system is different from its effect on the small axons with low Nf content of the visual system (Parhad et al., 1986). In the sciatic motor system, IDPN produces a severe $(90 \%)$ impairment in transport of $\mathrm{Nf}$ proteins, with a secondary impairment in the transport of other

\begin{tabular}{|c|c|c|c|c|c|}
\hline $\begin{array}{l}\text { Axon } \\
\text { type }\end{array}$ & $\mathrm{Nf} / \mu \mathrm{m}^{2}$ & $\mathrm{Mt} / \mu \mathrm{m}^{2}$ & $\%$ MOM & $\begin{array}{l}\mathrm{CSA} \\
\left(\mu \mathrm{m}^{2}\right)\end{array}$ & $\begin{array}{l}\text { Axons } \\
(n)\end{array}$ \\
\hline \multicolumn{6}{|l|}{ Proximal } \\
\hline $\begin{array}{l}\text { Control } \\
\text { IDPN }\end{array}$ & $74.1 \pm 7.2^{a}$ & $26.0 \pm 3.9$ & $5.2 \pm 1.8$ & $1.7 \pm 0.5$ & 8 \\
\hline $8^{b}$ & $142 \pm 27$ & $9.8 \pm 2.4$ & & $2.2 \pm 0.2$ & 3 \\
\hline 9 & $306 \pm 32$ & $9.2 \pm 3.2$ & & $1.9 \pm 0.5$ & 6 \\
\hline 12 & $377 \pm 14$ & $6.5 \pm 0.6$ & & $2.6 \pm 1.2$ & 5 \\
\hline 42 & $406 \pm 1.2$ & $13.3 \pm 2.1$ & $3.4 \pm 0.8$ & $10.3 \pm 3.3$ & 20 \\
\hline \multicolumn{6}{|l|}{ Distal } \\
\hline $\begin{array}{l}\text { Control } \\
\text { IDPN }\end{array}$ & $78.8 \pm 5.0$ & $36.2 \pm 2.9$ & $2.6 \pm 0.5$ & $1.9 \pm 0.21$ & 27 \\
\hline 8 & $118 \pm 10.8$ & $28.3 \pm 5.0$ & & $1.9 \pm 0.7$ & 5 \\
\hline 9 & $77.0 \pm 8.3$ & $34.6 \pm 3.1$ & $2.2 \pm 0.4$ & $1.6 \pm 0.2$ & 13 \\
\hline 12 & $26.8 \pm 5.9$ & $48.8 \pm 5.8$ & $4.2 \pm 1.1$ & $0.9 \pm 0.1$ & 10 \\
\hline 42 & $15.2 \pm 10.7$ & $37.9 \pm 5.0$ & $2.0 \pm 0.5$ & $1.3 \pm 0.1$ & 13 \\
\hline
\end{tabular}

${ }^{a}$ Means \pm SE given.

'Age of animals (in weeks).

cytoskeletal proteins (Griffin et al., 1978, 1985). In the visual system, it produces a moderate (50\%) impairment in the transport of Nf proteins without affecting the transport of other components of slow transport (Parhad et al., 1986). In addition, IDPN has a selective effect on the Nf-rich axons within a neuronal system. For example, in the visual system, large axons have a relatively greater accumulation of $\mathrm{Nf}$ proximally than do smaller axons (Fig. 7). Conversely, in the sciatic motor system, large fibers have a relatively greater loss of Nf distally than smaller fibers (Papasozomenos et al., 1981).

\section{Possible mechanisms for the decline in Nf content following IDPN intoxication}

IDPN reorganizes the cytoskeleton (Papasozomenos et al., 1981; Griffin et al., 1983) and impairs the transport of Nf along the entire length of the axon (Griffin et al., 1978; Parhad et al., 1986). This produces a marked accumulation of $\mathrm{Nf}$ in the most proximal part of the axon and a depletion of $\mathrm{Nf}$ in the rest of the axon (Fig. 3, Table 3). A uniform decrease in rate along the entire axon cannot explain the decline in Nf content, however. To produce a decline in Nf content within a segment of the nerve there must be a net decrease of $\mathrm{Nf}$ content within that segment. A sequential increment in the rate of transport of $\mathrm{Nf}$ along the axon could result in a progressive decline of $\mathrm{Nf}$ content throughout the axon. Such an increment in the rate of transport of $\mathrm{Nfs}$ could be produced if the Nfs in the more distal parts of the axon were less vulnerable to the effect of IDPN. In this regard it is relevant that $\mathrm{Nfs}$ become progressively more phosphorylated as they move distally (Nixon et al., 1982; Nixon and Logvinenko, 1986). These phosphorylated Nfs may be protected from the effect of IDPN. Alternative mechanisms for the decline of $\mathrm{Nf}$ content along the distal axon may include the degradation of the stagnant Nf within the axon proper. To date, Nf degradation within the axon proper has been described only in the disconnected distal segment following axotomy (Schlaepfer and Micko, 1978).

Whatever the mechanism for the decline in $\mathrm{Nf}$ content in IDPN model, it is distinct from that seen with regeneration. In 

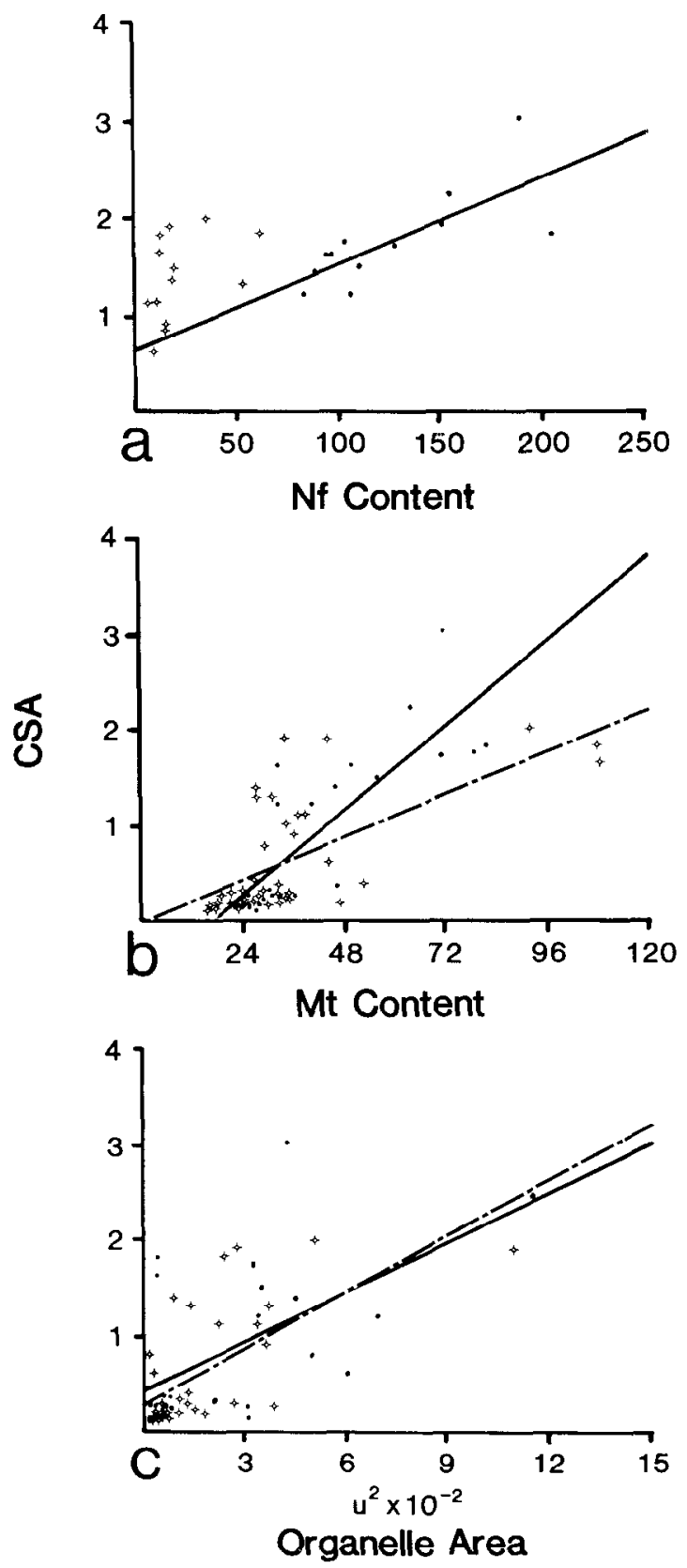

Figure 6. CSA of the distal axon as a function of (a) Nf content, (b) Mi content, and (c) area occupied by MOM in control $(\bullet,-)$ and chronically intoxicated $(\diamond,--)$ animals. Note that in $a$ there is a good correlation between Nf content and CSA in control $(r=0.73, p<0.02)$ but not following IDPN intoxication $(r=0.44, p>0.1)$; in $b$ there is a good correlation between Mt content and CSA in both control $(r=$ $0.84, p<0.001)$ and intoxicated animals $(r=0.66, p<0.001)$; in $c$ the correlation between area occupied by MOM and CSA is significant in both control $(r=0.55, p<0.01)$ and intoxicated $(r=0.67, p<$ $0.001)$ animals.

the latter case, there is a decrease in Nf content delivered to the axon. This results in atrophy that progresses somatofugally at a rate compatible with the rate of $\mathrm{Nf}$ transport (Hoffman et al., 1984, 1985b). In the IDPN model the Nf content is decreased simultaneously throughout the distal axon, and at a rate that is incompatible with that of $\mathrm{Nf}$ transport.

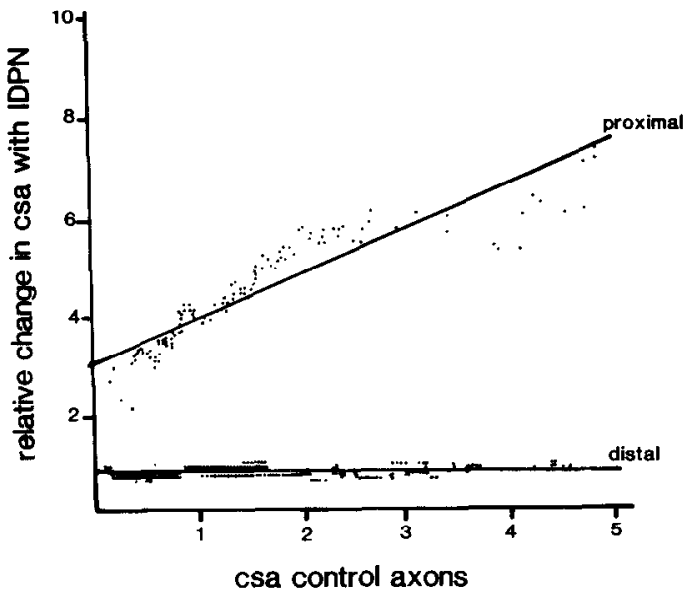

Figure 7. Relative change in CSA following IDPN intoxication as a function of (preintoxication) axonal caliber. The preintoxication axonal CSAs are obtained from the 42-week-old control animal. The relative change in CSAs is the ratio of CSAs of 42-week-old IDPN animals/ CSAs in 42-week-old controls. These data are graphed with the largest (control) axons corresponding to the largest axons following IDPN intoxication. In the proximal axons there is a direct and linear correlation between the relative increase in CSA and the preintoxication CSA (slope: $y=3.03+0.87 x ; n=200 ; r=0.91, p<0.001)$. In the distal regions, all axons irrespective of size atrophy to the same degree following intoxication (slope: $y=0.69-0.007 x, n=400 ; r=0.07, p>0.05$ ).

\section{Nf density, content, and CSA following IDPN intoxication}

Following the alteration in Nf transport produced by IDPN, a wide range of $\mathrm{Nf}$ densities is obtained, from $400 \mathrm{Nf} / \mu \mathrm{m}^{2}$ in the proximal axon to $15 \mathrm{Nf} / \mu \mathrm{m}^{2}$ in the distal axon. The inter-Nf distance, however, changes only minimally. These seemingly paradoxical results can be explained by the fact that $\mathrm{Nf}$ profiles are grouped (Schlaepfer, 1983; Fig. 2b). A change in Nf content affects the $\mathrm{N}$-free area between the groups, thus affecting the $\mathrm{Nf}$ density but not the inter-NF distance within a group.

In the proximal axon, a direct, nonlinear correlation exists between Nf content and CSA. Soon after intoxication, the increase in Nf content exceeds that of the CSA, resulting in a rapid and marked rise in $\mathrm{Nf}$ density. Once the $\mathrm{Nf}$ density reaches a new constant of approximately $400 \mathrm{Nf} / \mu \mathrm{m}^{2}$, the axonal CSA increases linearly with the increase in Nf content (Fig. 3a, Table 3 ). These results suggest that the packing density of $\mathrm{Nf}$ reaches a maximum when the Nf groups become confluent, and the Nfs maintain a minimum distance between each other. Any additional increase in Nf content is accommodated by a proportional increase in the CSA of axons.

In the distal axon, on the other hand, there is no correlation between the reduction in Nf content and axonal atrophy (Fig. $6 a$ ). There is a marked and continuous loss of Nf content and density but only a minor and static change in the CSA, and no change in axonal shape is seen. This is distinct from the atrophy seen in the sciatic motor fibers following IDPN (Griffin et al., unpublished observations) or axotomy (Hoffman et al., 1984, $1985 \mathrm{~b}$ ). In the latter 2 situations, the Nf density remains normal, and the CSA decreases markedly in proportion to the decrease in Nf content. In addition, these axons change their shape, becoming less circular. These results suggest that small axons are to a large extent able to maintain CSA and shape in the face of marked decrease in the Nf content, and factors other than Nf content must be capable of influencing size and shape of these axons. 


\section{Role of other factors in determining CSA of small axons}

Mts and MOM play significant roles in determining volume and shape of axons and dendrites in certain developing and adult systems (Friede and Samorajski, 1970; Friede et al., 1971; Smith, 1973; Nadelhaft, 1974; Sasaki et al., 1983; Sasaki-Sherrington et al., 1984). The Mt content (Friede and Samorajski, 1970; Friede et al., 1971; Fig. $6 b$ ) and the area occupied by MOM (Fig. 6c) increase with an increase in CSA of normal axons. These relationships are not altered significantly following IDPN intoxication. Thus, following the depletion of Nfs, the Mts and MOM are in part responsible for maintaining axonal caliber.

Axonal caliber may be influenced not only by the total number of the cytoskeletal elements but also by their position and distribution (Sasaki-Sherrington et al., 1984). For example, in the IDPN model there is an early segregation of Nfs from Mts. Axonal atrophy in the distal segment is seen concurrently with this reorganization of the cytoskeleton and correlates best with this change.

Factors that are not visible by conventional ultrastructural methods may contribute to the volume necessary to maintain the size and shape of neuronal processes (Ellisman and Porter, 1983; Sasaki-Sherrington et al., 1984). The source of this volume is not known, but it could be associated with soluble axonal contents, subaxolemmal cytoskeleton including actin and its membrane-linking proteins, and the microtrabecular lattice.

\section{Summary and conclusions}

This model provides insight into the factors that maintain axonal caliber when $\mathrm{Nf}$ content is altered. It has the advantages of an agent that produces a selective defect in transport of Nfs and a system with axons of a critical size in which Nfs, Mts, and MOM each have a noticeable effect on axonal caliber. Certain conclusions can be drawn from this model; these include:

1. In small axons, Mts and MOM can take over the structural role of Nfs when the latter are depleted. In these axons, Nfs do not play as important a role in maintaining caliber as they do in large fibers.

2. Nf density can be altered drastically without affecting the inter-Nf distance.

3. The position of cytoskeletal elements and organelles to each other may contribute to changes in shape and volume. For example, the atrophy seen in this model correlated temporally with a reorganization of the cytoskeletal elements, and may be due to it.

4. The decrease in Nf content seen in the distal axons in this model can best be explained by a nonuniform impairment in the rate of transport of $\mathrm{Nfs}$, resulting in faster, less impaired rates distally.

\section{References}

Berthold, C. H. (1978) Morphology of normal peripheral axons. In Physiology and Pathology of Axons, S. G. Waxman, ed., pp. 3-63, Raven, New York.

Black, M. M., and R. J. Lasek (1980) Slow components of axonal transport: Two cytoskeletal networks. J. Cell Biol. 86: 616-623.

Clark, A. W., J. W. Grittin, and D. L. Price (1980) The axonal pathology in chronic IDPN intoxication. J. Neuropathol. Exp. Neurol. 39: 42-55.

Duncan, D. B. (1955) Multiple range and multiple F tests. Biometrics 11: $1-42$.

Dyck, P. J., A. Lais, J. Karnes, M. Sparks, and P. J. B. Dyck (1985) Peripheral axotomy induces neurofilament decrease, atrophy, de- myelination and degeneration of root and fasciculus fibers. Brain Res. 340: 19-36.

Ellisman, M. H., and K. R. Porter (1983) Introduction to the cytoskeleton. In Neurofllaments, C. A. Marotta, ed., pp. 3-27, University of Minnesota Press, Minneapolis, MN

Friede, R. L., and T. Samorajski (1970) Axon caliber related to neurofilaments and microtubules in sciatic nerve fibers of rats and mice. Anat. Rec. 167: 379-388.

Friede, R. L., T. Miyaghishi, and K. H. Hu (1971) Axon caliber, neurofilaments, microtubules, sheath thickness and cholesterol in cat optic nerve fibers. Anat. Rec. 108: 365-373.

Griffin, J. W., P. N. Hoffman, A. W. Clark, P. T. Carrol, and D. L. Price (1978) Slow axonal transport of neurofilament proteins: Impairment by $\beta, \beta^{\prime}$-iminodipropionitrile administration. Science 202 : 633-635.

Griffin, J. W., K. E. Fahnestock, D. L. Price, and P. N. Hoffman (1983) Microtubule-neurofilament segregation produced by $\beta, \beta^{\prime}$-iminodipropionitrile: Evidence for the association of fast axonal transport with microtubules. J. Neurosci. 3: 557-566.

Griffin, J. W., I. M. Parhad, B. Gold, D. Price, P. Hoffman, and K. Fahnestock (1985) Axonal transport of neurofilament proteins in IDPN neurotoxicity. Neurotoxicology 6: 43-54.

Hoffman, P. N., and R. J. Lasek (1975) The slow component of axonal transport. Identification of major structural polypeptides of the axon and their generality among mammalian neurons. J. Cell Biol. 66. 351-366.

Hoffman, P. N., J. W. Griffin, and D. L. Price (1984) Control of axonal caliber by neurofilament transport. J. Cell Biol. 99: 705-714.

Hoffman, P. N., J. W. Griffin, B. G. Gold, and D. L. Price (1985a) Slowing of neurofilament transport and the radial growth of developing nerve fibers. J. Neurosci. 5: 2920-2929.

Hoffman, P. N., G. W. Thompson, J. W. Griffin, and D. L. Price (1985b) Changes in neurofilament transport coincide temporally with alterations in the caliber of axons in regenerating motor fibers. J. Cell Biol 101: $1332-1340$

Kleinbaum, D. G., and L. L. Kupper (1978) Applied Regression Analysis and Other Multivariable Methods, pp. 100-106, Duxbury Press, North Scituate, MA.

Lasek, R. J., M. M. Oblinger, and P. F. Drake (1984) Molecular biology of neuronal geometry: Expression of neurofilament genes influences axonal diameter. Cold Spring Harbor Symp. Quant. Biol. 48: 731744.

Nadelhaft, I. (1974) Microtubule densities and total numbers in selected axon of the crayfish abdominal nerve cord. J. Neurocytol. 3 73-86.

Nixon, R. A., and K. B. Logvinenko (1986) Multiple fates of newly synthesized neurofilament proteins: Evidence for a stationary neurofilament network distributed non-uniformly along axons of retinal ganglion cell neurons. J. Cell Biol. 102: 647-659.

Nixon, R. A., B. A. Brown, and C. A. Marotta (1982) Posttranslational modification of a neurofilament protein during axoplasmic transport: Implications for regional specialization of CNS axons. J. Cell Biol. 94: $150-158$.

Papasozomenos, S. C., L. Autilio-Gambetti, and P. Gambetti (1981) Reorganization of axoplasmic organelles following $\beta, \beta^{\prime}$-iminodipropionitrile administration. J. Cell Biol. 91: 866-871.

Parhad, I. M., J. W. Griffin, D. L. Price, A. W. Clark, L. C. Cork, N. R. Miller, and P. N. Hoffman (1982) Intoxication with $\beta, \beta^{\prime}$-iminodipropionitrile: A model of optic disc swelling. Lab. Invest. 46: 186195.

Parhad, I. M., J. W. Griffin, P. N. Hoffman, and J. F. Koves (1986) Selective interruption of axonal transport of neurofilament proteins in the visual system by $\beta, \beta^{\prime}$-iminodipropionitrile (IDPN) intoxication. Brain Res. 363: 315-324.

Roots, B. I. (1983) Neurofilament accumulation induced in synapses by leupeptin. Science 221: 971-972.

Sasaki, S. E., J. K. Stevens, and N. Bodick (1983) Serial reconstruction of microtubular arrays within dendrites of the cat retinal ganglion cell: The cytoskeleton of a vertebrate dendrite. Brain Res. 259:193206.

Sasaki-Sherrington, S. E., R. Jacobs, and J. K. Stevens (1984) Intracellular control of axial shape in non-uniform neurites: A serial electron microscopic analysis of organelles and microtubules in A1 and A1 1 retinal amacrine neurites. J. Cell Biol. 98: 1279-1290 
Schlaepfer, W. W. (1983) Neurofilaments of mammalian peripheral nerve. In Neurofilaments, C. A. Marotta, ed., pp. 57-85, University of Minnesota Press, Minneapolis, MN.

Schlaepfer, W. W., and S. Micko (1978) Chemical and structural changes of neurofilaments in transected rat sciatic nerve. J. Cell Biol. 78:369378.
Smith, R. S. (1973) Microtubule and neurofilament densities in amphibian spinal root nerve fibers: Relationship to axoplasmic transport. Can. J. Physiol. 51: 798-806.

Snedecor, G. W., and W. G. Cochran (1980) Statistical Methods, p. 185, The Iowa State University Press, Ames, IA. 